\title{
NOMINATA
}

\section{CONSULTORES AD HOC 2013}

Volume 8, NúmERos 13 e 14

1. ADMAR ALMEIDA DA COSTA - UFRR

2. AgNALdo CUOCO PORTUGAL - UNB

3. AlEX GONÇALVES MUNIZ - UFU/IFBA

4. Altair AlBERTo FÁVERo - UPF

5. ANA LÚCIA GALINKIN $-U N B$

6. ANDRÉ LUIZ CAES - UEG

7. ANTÔNIO ALVES DE MELO - IFITEPS

8. BETINA RIBEIRO RODRIGUES DA CUNHA - UFU

9. CARLOS ARTHUR RIBEIRO DO NASCIMENTO - PUC-SP

10. CARLOS FREDERICO BARBOZA - PUC MINAS

11. CeCília Cintra Cavaleiro de Macedo - PUC-SP

12. DEYVE REDYSON MELO DOS SANTOS - UFP

13. DULCINA TERESA BONATI BORGES $-U F U / F C U$

14. EDILSON BALTHAZAR BERREIRA JÚNIOR - FAMETRO

15. EDLA EGGERT - UNISINOS

16. EUNICE SIMÕES GOMES - UFPB

17. Flávio AUgusto SENRA - PUC-MINAS

18. FRANKLIN LEOPOLDO E SILVA - USP/FACULDADE DE SÃO BENTO -SP

19. GILBRAZ ARAGÃO - UNICAP

20. GLÓRIA NEVES DUTRA ESCARIÃO - UFPB

21. HARLEY JULIANO MANTOVANI - FCU

22. HAROLDO REIMER - PUC-GO

23. IRENE DE ARAÚJO - UFRN

24. IRINÉIA FRANCO - UFAL

25. JAQUELINE ZIROLDO DOLGHIE - MACKENZIE

26. JOACHIM ANDRADE - FCU

27. JOÃO CARLOS ALMEIDA - FACULDADE DEHONIANA

28. JOÃO MAC DOWELL - FAJE

29. JOEL ANTÔNIO FERREIRA - PUC-GO

3o. JONAS ROOS - UFJF

31. JOSÉ AMÉRICO DE LACERDA JÚNIOR - UFF

32. JOSÉ BENEDITO DE ALMEIDA JÚNIOR - UFU 
33. JosÉ CARLOS BRUNI - USP/FACULDADE DE SÃO BENTO -SP

34. JÚNIOR VASCONCELOS DO AMARAL - FAJE

35. KARINA BELLOTI - UFPR

36. LÍlIAN WURZBA IOSHIMOTO - IJEP

37. LURDES CARON - UNIPLAC

38. MARCEL MANO - UFU

39. MARCIANO AdÍlio SPICA - UNICENTRO

4O. Marcio Gimenes de PaUla - $U N B$

41. MARCELO NEVES OP - ANGELICUM - ROMA

42. MARCOS CÉSAR SENEDA - UFU

43. MARIA CRISTINA MARIANTE GUARNIERI - PUC-SP

44. MARIA MaURA DE MORAES - FCU

45. MARIANA LEAL BARROS - USP

46. MARY RUTE ESPERANDIO - PUC-PR

47. OlaVo P. CALÁBRIA - UFU

48. PAUlo SÉRgIO DE PROENÇA - FATIPI

49. RICARDO MARIANO - PUCRS/USP

5o. ROBERLEI PANASIEWICZ - PUC MINAS

51. ROBERT DAIBERT - UFJF

52. ROBERTO PEREIRA MIGUEL - PUC-SP

53. RODRIGO LEISTNER - UNISINOS

54. ROSA GITANA MENEGHETTI - UNIMEP

55. RUbENS GARCIA NUNES SOBRINHO - UFU

56. SÉrgio DE SIQUEIRA CAMARGO - FCU

57. SÉrgio Rogério AZEVEdo JUNQUEIRA - PUC-PR

58. SUZANA GOMES - UFMG

59. VALMOR DA SILVA - PUC-GO

6o. VANI TEREZINHA DE REZENDE - USP/FCU

61. VÂNIA NORONHA - PUC MINAS

62. VIVIANE CRISTINA CÂNDIDO - UFPB

63. WELLINGTON TEODORO DA SILVA - PUC MINAS

64. WILLIAN DE SOUZA PEREIRA - UFMT 